\title{
Las externalidades de la explotación de minerales en la agenda del gobierno brasileño: Análisis a partir del modelo de múltiple streams
}

\author{
Carmen Pineda Nebot ${ }^{1}$, Jones Barros ${ }^{2}$
}

Recibido: 09/01/2020 / Aceptado: 13/03/2020

Resumen. La preservación del medio ambiente a escala global siempre estuvo en el orden del día de las discusiones debido a la necesidad de un modelo de desarrollo económico sostenible. Este trabajo pretende comprobar a partir de qué momento la cuestión de los embalses de desechos de minerales pasa a colocarse en la agenda de los gobiernos. Para ello, se realiza una revisión documental y un análisis del proceso de formulación de la política pública basado en el modelo de Múltiple Streams (Modelo de Múltiples Flujos), desarrollado por Kingdon (2003). El argumento desarrollado es que se abrió una "ventana de oportunidad", por la convergencia de los flujos (problemas, soluciones, política), como resultado de la rotura de un embalse en el municipio de Brumadinho, en el Estado de Minas Gerais.

Palabras clave: Seguridad de embalses; política pública; agenda del gobierno.

The externalities of the exploitation of minerals in the brazilian government agenda: analysis from the multiple streams model

\begin{abstract}
The preservation of the environment on a global scale was always on the agenda of the discussions due to the need for a model of sustainable economic development. This work aims to verify from what moment the issue of mineral waste reservoirs is placed on the government's agenda. For this, a documentary review and an analysis of the public policy formulation process based on the Multiple Streams model, developed by Kingdon (2003). The argument developed is that a "window of opportunity" was opened, due to the convergence of flows (problems, solutions, politics), as a result of the breakage of a reservoir in the municipality of Brumadinho, in the state of Minas Gerais.
\end{abstract}

Keywords: Reservoir safety; public politics; government agenda.

Sumario. 1. Introducción. 2. La política pública como acción de gobierno. 2.1. La Política Nacional de Seguridad de los embalses en Brasil. 3. Aspectos metodológicos de la investigación. 3.1. El problema de los embalses de desechos y su análisis a partir del modelo de múltiple streams. 3.1.1. El problema. 3.1.2. Las soluciones. 3.1.3. La política. 3.1.4. Oportunidad de cambio a partir de la convergencia de los tres flujos: construcción de la agenda. 3.1.5. Convergencia entre problemas, soluciones y dinámica política: las externalidades de la explotación de minerales en la agenda gubernamental en Brasil. 4. Resultados y discusiones. 4.1. Las soluciones del Gobierno para afrontar el problema. 4.1.1. El Decreto $\mathrm{n}^{\circ}$ 9.691, de 25 de enero de 2019. 4.1.2. Resolución $\mathrm{n}^{\circ} 1$, de 28 de enero de 2019. 4.1.3. Resolución $\mathrm{n}^{\circ} 2$, de 28 de enero de 2019. 5. Conclusiones.

Cómo citar: Pineda Nebot, C.; Barros, J. (2020): Las externalidades de la explotación de minerales en la agenda del gobierno brasileño: Análisis a partir del modelo de múltiple streams, en Cuadernos de Gobierno y Administración Pública 7-2, 75-84.

\section{Introducción}

Considerado por los participantes como uno de los eventos mundiales más importantes, la Conferencia Rio +10 , realizada del 26 de mayo al 29 de agosto de 2002, en Johannnesburgo (Sudáfrica), contó con representantes de gobiernos de más de 150 países que se reunieron para discutir el medio ambiente global y las necesidades de desarrollo.

En su documento final, la Conferencia consideró la extracción de minerales como una actividad fundamental para el desarrollo económico y social de muchos países, teniendo en cuenta que los minerales son esenciales para la vida moderna. No obstante, el respeto a 
los derechos humanos básicos, la protección del medio ambiente y la utilización equilibrada de los recursos naturales también son puntos importantes del documento y en él se señala el desafío que tienen las naciones para impulsar un desarrollo sostenible.

En Brasil, la actividad de explotación de minerales se remonta al período colonial y mantiene su fuerza hasta el siglo XXI. Unida a la industria extractivista, representa el 4\% del Producto Interior Bruto (PIB) y contribuye al 25\% del saldo comercial brasileño, según el Ministerio de Minas y Energía. En 2017 se exportaron minerales por un valor de 46,4 billones de dólares, con un superávit de 23,4 billones de dólares (Ibram/2018). En el sector reina la empresa Vale, fundada en 1942 y privatizada en 1997, que es la mayor productora de mineral de hierro del mundo, siendo su poder inmenso ${ }^{3}$.

Aunque las externalidades provocadas por el desarrollo de la actividad son evidentes y crean conflictos en las comunidades locales. Entre las más graves está la rotura de los embalses de desechos de minerales en Mariana y Brumadinho, dos municipios del estado de Minas Gerais. La rotura, en noviembre de 2015, de la presa de Samarco $^{4}$ en el municipio de Mariana ${ }^{5}$ provocó enormes daños ambientales y la muerte de varios trabajadores y vecinos del entorno (19 personas). Arrasó dos ciudades y recorrió más de 600 kilómetros hasta el Atlántico ${ }^{6}$, alterando las vidas de cientos de miles de personas y dañando gravemente el ecosistema (Kokke, 2017). Siendo considerado el mayor desastre ambiental de Brasil y uno de los mayores del mundo, al haber llegado a los cuerpos hídricos y al medio ambiente unos treinta y cuatro millones de metros cúbicos de desechos (Silva et al., 2017). En junio de 2018 la empresa Vale decidió, después de firmar un acuerdo con las fiscalías, las defensorías y las abogacías del Estado brasileño correspondientes al Estado central y a los estados de Minas Gerais y Espírito Santo afectados por la catástrofe, contribuir con alrededor de 557,5 millones de reales (144 millones de dólares) a la restauración del medio ambiente y de las comunidades afectadas por la ruptura de la balsa.

Pero cuatro años después, en marzo de 2019, otro de los embalses de residuos de minerales de la empresa Vale, una de las mayores multinacionales brasileñas, colapsó y volvió a provocar un gran desastre. En este caso con la muerte de 235 personas y la desaparición de otras 35 , además de provocar importantes daños ambientales en la zona. En este caso fueron 13 millones de metros

La empresa Vale tiene 55.000 empleados en Brasil y una facturación de 36.500 millones de dólares (32.500 millones de euros).

4 La presa fue construida para acoger los residuos provenientes de la extracción del mineral de hierro que es retirado de diversas minas de la región (O Globo, 2015).

5 Para conocer algo más sobre el tema ver: "Desastre no Vale do Rio Doce" (Milanez y Losekann, 2016), organizado por el PoEMA/ Organon; "Desastre na Bacia do Rio Doce" (Losekann y Mayorga, 2018), elaborado por la Organización Interinstitucional Mariana Rio Doce y Organon; y "Mineração, Violência e Resistências" (Zhouri, 2017), de la Asociación Brasileña de Antropología.

6 Los contaminantes, compuestos principalmente por óxido de hierro y sílice, recorrieron directamente $55 \mathrm{~km}$ en el Rio Gualaxo do Norte, avanzando en el río de Carmo y otros $22 \mathrm{~km}$ hasta el río Doce. La tragedia socioambiental se extendió a lo largo de 663,2 km de cursos de agua, alcanzando hasta el litoral del Estado de Espírito Santo (Kokke, 2017). cúbicos de lodo los que avanzaron a 80 kilómetros por hora en algunos puntos, destruyendo casas, y llegando al río. Resulta asombroso constatar que, más de tres años después, Brasil siga debatiendo sobre los mismos problemas que ocasionaron la primera tragedia. Más aún, que, durante todo este tiempo, nada se haya hecho para mejorar la seguridad de este tipo de instalaciones.

Según ANA (Agencia Nacional del Agua) existen 790 embalses de desechos de minerales de un total de más de 24 mil embalses en Brasil, la mayoría de ellos de pequeño tamaño. De ellos 417 están incluidos en la Política Nacional de Seguridad de Embalses (PNSB), pero 369 no lo están. Todos los embalses están dispuestos por categorías basadas en el riesgo de rotura y en el potencial impacto que causaría, en el caso de accidente, en las comunidades próximas y en el medio ambiente.

El artículo pretende verificar a partir de qué momento la cuestión de los embalses de desechos de minerales pasa a colocarse en la agenda de los gobiernos y a institucionalizarse por las normas nacionales, del nivel macro al micro. El estudio utiliza como referencia la teoría del ciclo de políticas públicas para por medio de la revisión documental de la política minera en Brasil, realizar un análisis del proceso de formulación de la política pública basado en el modelo de Multiple Streams (Modelo de Múltiples Flujos), desarrollado por Kingdon (2003), focalizando la decisión gubernamental en el cambio del sistema de control de los embalses de desechos en todo Brasil.

El texto está estructurado en tres secciones además de la introducción y las consideraciones finales. En la primera sección se discute la política pública como acción de gobierno y se trata la Política Nacional de Seguridad de los embalses en Brasil. La segunda sección aborda los aspectos metodológicos de la investigación, analiza el problema de los embalses de desechos y su análisis a partir del modelo de multiple streams, examina y describe los documentos oficiales creados por el Gobierno, que establecieron directrices para solucionar los problemas. Los resultados de la investigación se presentan en la tercera sección.

\section{La política pública como acción de gobierno}

Existe una variedad de definiciones sobre el tema políticas públicas, lo que hace posible una diversidad de conceptos y definiciones al respecto. Para Souza (2006), el campo de conocimiento de las políticas públicas permite analizar al gobierno en acción y proponer cambios en el rumbo o curso de esas acciones. Su formulación constituye una fase en la que los gobiernos democráticos traducen sus propósitos y plataformas electorales en programas y acciones que producirán resultados o cambios en el mundo real.

La política pública, también es presentada como campo multidisciplinar, siendo una forma de construir un campo común de discusión entre diversas perspectivas que permitan el conocimiento sobe las acciones del Estado (Marques, 2013; Faria, 2010). 
Para una mejor comprensión se trató de analizar la temática sobre políticas públicas a partir de dos corrientes, americana y europea, presentes en la literatura.

La corriente americana trata del estudio de la política enfocada en el análisis de la acción de los gobiernos. Opuesta a la americana, la corriente europea pone el énfasis en los estudios e investigaciones enfocados en el análisis sobre el Estado y sus instituciones (Souza, 2006).

Entre los principales fundadores de los estudios sobre políticas públicas destacan los americanos: Simon (1947) y Lindblom (1979). Simon (1947) introdujo el concepto de racionalidad limitada de los decisores públicos (policymakers) y argumenta que la preocupación central de la teoría administrativa debe ser la racionalidad de las decisiones y su adecuación para la realización de objetivos específicos, ya que existen límites para la racionalidad humana.

Lindblom (1979) propuso la incorporación de otras variables a la formulación y al análisis de políticas públicas, como las relaciones de poder y la integración entre las diferentes fases del proceso de decisión, así como el papel de las elecciones, de las burocracias, de los partidos y de los grupos de interés.

Las decisiones sobre las políticas deberían seguir los procedimientos técnicos (Simon, 1947), hasta el punto que para Lindblom (1979), las políticas son resultado también de un contexto real en el que los partidos políticos y los grupos de intereses actúan para influir en la toma de decisiones, haciendo que al final prevalezca lo que para ellos es conveniente.

La comprensión sobre las políticas públicas presentada por los autores Simon (1947) y Lindblom (1979), abarcan el proceso de formulación e implementación de las políticas públicas, lo cual debería ser visto como un proceso, y no como un producto técnico solamente.

En Brasil, los estudios sobre políticas públicas están influidos por los teóricos americanos, cuya acción de los gobiernos ha sido el foco de los estudios, en las más diversas áreas como la educación y la salud. Sin embargo, el foco del estudio propuesto este puesto en el papel del Estado y sus instituciones, por entender que es por medio de estos que los gobiernos buscan soluciones para los problemas públicos, siendo un planteamiento que desde el 2000 va ganando fuerza en Brasil (Metten et al., 2015).

\subsection{La Política Nacional de Seguridad de los embalses en Brasil}

En Brasil, la Política Nacional de Seguridad de los Embalses (PNSB) y el Sistema Nacional de Información sobre Seguridad de los Embalses (SNISB) están regulados por la Ley n ${ }^{\circ} 12.334$, de 20 de septiembre de 2010. Esta Ley se aplica a los embalses destinados a la acumulación de agua para cualquier uso, en la disposición final habla también de la acumulación temporal de desechos y de la acumulación de residuos industriales. En el análisis de la Ley 12.334, se verifica que los objetivos dan PNSB están relacionados intrínsecamente a la cuestión de la seguridad, hecho que hace deducir que la construcción de embalses implica un riesgo ambiental y social, especialmente cuando están cerca del nacimiento de un rio, el más implementado por las empresas mineras en Brasil.

Las cuestiones de seguridad de los embalses tratadas en los objetivos de la PNSB están relacionadas a los fundamentos y a la fiscalización y reivindica que está debe ser contemplada en las fases de planificación, proyecto, construcción, primer llenado y primer desagüe, operación, desactivación y usos futuros. La evaluación de seguridad de los embalses se debe hacer por medio de inspecciones periódicas para la identificación de anomalías estructurales y funcionales del dique y de sus estructuras auxiliares. Esa evaluación es el principal instrumento para la auditoría y la prevención de posibles accidentes, aunque, solo es eficaz si está acompañada de test regulares para asegurar el comportamiento correcto y el buen funcionamiento, entre otros, de los sensores de presión internos, estáticos e hidráulicos del embalse - los "piezómetros". También son necesarias auditorias independientes para asegurar que los instrumentos están funcionando y los datos generados son dignos de confianza y representativos de la situación real del dique.

La responsabilidad de los diversos actores públicos (Unión, Estados y Municipios) y de las empresas es imprescindible para que los objetivos de la PNSB sean efectivos y garanticen calidad de vida socioambiental y eviten tragedias como las ocurridas en Mariana y Brumadinho (MG).

La PNSB también hace referencia a la importancia de la promoción de mecanismos de participación y control social, así como de la publicidad (Tenório, 1997) y del estímulo a participar, directa o indirectamente, de las acciones preventivas y de emergencia. Sin embargo, informaciones divulgadas por los medios de comunicación revelan que los datos técnicos producidos por los instrumentos (Artículo $6^{\circ}$ ) de la PNSB no eran hechos públicos o estaban basados en informes de consultoras contratadas por las propias empresas, con posible ocultación de la realidad de estos embalses.

La PNSB establece los instrumentos para garantizar la seguridad de los embalses del país, que son: i) el sistema de clasificación de embalses por categoría de riesgo y por daño potencial asociado; ii) el Plan de Seguridad de Embalses; iii) el Sistema Nacional de Información sobre Seguridad de Embalses (SNISB); iv) el Sistema Nacional de Información sobre el Medio Ambiente (Sinima); v) el Registro Técnico Federal de Actividades e Instrumentos de Defensa Ambiental; vi) el Registro Técnico Federal de Actividades Potencialmente Contaminantes o Utilizadoras de Recursos Ambientales; vii) el Informe de Seguridad de Embalses.

Veesaert et al. (2005) destacan que la cuestión de la seguridad de los embalses en Brasil se lleva discutiendo desde 2003 pues no existía una ley específica que tratase la cuestión, hecho que revela la falta de atención de las autoridades competentes, teniendo en cuenta la existencia de un gran número de embalses, especialmente de embalses de desechos de minerales, a causa de la intensa 
explotación de esa actividad en el país. También señala que desde 2003 se comenzó a tramitar en el Senado Federal el Proyecto de Ley 1.181 de 2003, el cual trataba de la Responsabilidad Legal, que implicaba al propietario del embalse y al ingeniero responsable de la seguridad. En 2004, el Proyecto 4.038 sugería la contratación de un seguro obligatorio contra la rotura de embalses. $\mathrm{Al}$ examinar la Ley 12.334, de 20 de septiembre de 2010, se ve que no se hace mención a las sugerencias que se planteaban en los dos proyectos de ley analizados por Veesaert et al. (2005), aunque sean cuestiones muy importantes para garantizar la vida de los trabajadores y de las comunidades del entorno de los grandes proyectos instalados en Brasil.

La PNSB dispone además que la Agencia Nacional de Aguas (ANA) sea la responsable de organizar, implantar y gestionar el Sistema Nacional de Información sobre Seguridad de los Embalses (SNISB); promover la articulación entre los órganos fiscalizadores de los embalses; coordinar la elaboración del Informe de Seguridad de Embalses; y recibir denuncias de los demás órganos o entidades fiscalizadores sobre cualquier no conformidad que implique un riesgo inmediato para la seguridad o cualquier accidente ocurrido en los embalses.

Brasil posee, en estos momentos, un registro con 24.092 embalses con diferentes finalidades, como acumulación de agua, de desechos mineros o industriales y para la producción de energía, que han sido censados por 31 órganos fiscalizadores. Buena parte, 9.827 (el 41\%), son embalses de riesgo. De ellos, en el Informe de Seguridad de los Embalses de 2017, 3.545 fueron clasificados en la Categoría de Riesgo (CRI), 5.459 de Daño Potencial Asociado (DPA) y 723 (el 3\%) fueron clasificados simultáneamente como de CRI y DPA altos.

Después del accidente de Mariana se presentaron varios proyectos para modificar la ley, con el fin de endurecer las medidas de seguimiento y control. Se pretendía, entre otras cosas, que aquellos embalses con mayor riesgo fueran inspeccionados inmediatamente. Ya que como señaló Andressa Lanchoti coordinadora del equipo de fiscales de Minas Gerais y de la policía federal que investigó lo qué ocurrió en Brumadinho. "Necesitamos un cambio de comportamiento muy importante para recuperar la confianza porque en menos de tres años hubo dos desastres implicando a la misma empresa (Vale) en dos minas que tenían certificado de estabilidad y una auditoría externa. Y ambas colapsaron. Eso supone una falta de credibilidad, no solo de Vale, sino de todo el sistema brasileño de gestión de balsas". Lanchoti también indicó que el Estado debería asumir la fiscalización que ahora es más bien un autocontrol de las empresas y aumentar el número de inspectores, que en ese momento era de 34 para todo Brasil cuando solo Minas Gerais es mayor que toda España.

Hay que señalar, porque es importante, que ANA preguntó, para redactar el Informe de Seguridad de los Embalses de 2017, al órgano fiscalizador del embalse de Brumadinho si éste estaba en situación crítica, a lo que la Agencia Nacional de Minas (ANM), responsable de la fiscalización, contesto que no.

\section{Aspectos metodológicos de la investigación}

Se trata de una investigación cualitativa de carácter exploratoria descriptiva. Como instrumentos de recogida de datos fueron utilizados documentos de dominio público. Los documentos examinados y descritos fueron: Ley $\mathrm{n}^{\circ} 12.334$, de 20 de septiembre de 2010 , decreto $\mathrm{n}^{\circ}$ 9.691 , de 25 de enero de 2019 , resolución $\mathrm{n}^{\circ} 1$, de 28 de enero de 2019, resolución $\mathrm{n}^{\circ} 2$, de 28 de enero de 2019.

Los análisis del proceso de formulación de la política pública están basados en el modelo de Multiple Streams (Modelo de Múltiples Flujos), desarrollado por Kingdon (2003). El estudio presta atención a los momentos de pre-decisión y decisión gubernamental acerca del cambio en el sistema de control de los embalses de desechos en Brasil, lo que justifica la elección de dicho modelo por comprender que el mismo hace posible el análisis de los momentos de la política pública.

\subsection{El problema de los embalses de desechos y su análisis a partir del modelo de múltiple streams}

¿Por qué algunos problemas se convierten en importantes para un gobierno? ¿Cómo se incluye una idea en el conjunto de preocupaciones de los formuladores de las políticas y se transforman en políticas públicas? Esas cuestiones constituyen el eje central de "Agendas, alternatives and public policies" de Kingdon (2003).

Kingdon (2003) considera que una agenda gubernamental es una relación de temas que son objeto de atención de los gobiernos y de los funcionarios en un determinado momento histórico. Sus ideas, aunque resultado del análisis sobre las políticas del gobierno americano en el área de salud y de transporte pueden ser aplicadas a estudios diversos de agendas especializadas como los diques de desechos de minerales u otros. Kingdon (2003) entiende las políticas públicas como conjuntos formados por cuatro procesos: i) establecimiento de una agenda de políticas públicas; ii) formación de alternativas para la formulación de políticas públicas, de modo que puedan ser hechas elecciones; iii) elección dominante entre un conjunto de alternativas disponibles; iv) implementación de la decisión.

Hay que destacar que el análisis realizado en este trabajo con el modelo de Kindgon (2003) solo tendrá en cuenta los dos primeros procesos de los cuatro presentados, los denominados de pre-decisión. Prestando especial atención al momento de construcción de la agenda da política pública. El modelo elaborado por John W. Kingdon (2003) es considerado adecuado para permitir el análisis de los momentos de "pre-decisión" y de "decisión", o sea, la selección de los problemas y la elección de las alternativas de políticas que se eligen como objeto del proceso de decisión.

En su búsqueda por comprender como algunas cuestiones pasan a integrar la agenda y a ser consideradas por los formuladores de políticas, Kingdon (2003) al estudiar el gobierno norteamericano las denomina como una "anarquía organizada", la cual está influida por tres flujos de decisión: problemas (problems), soluciones o alternativas (policies) y política (politics). 
Figura 1. Modelo desarrollado por Kingdon.

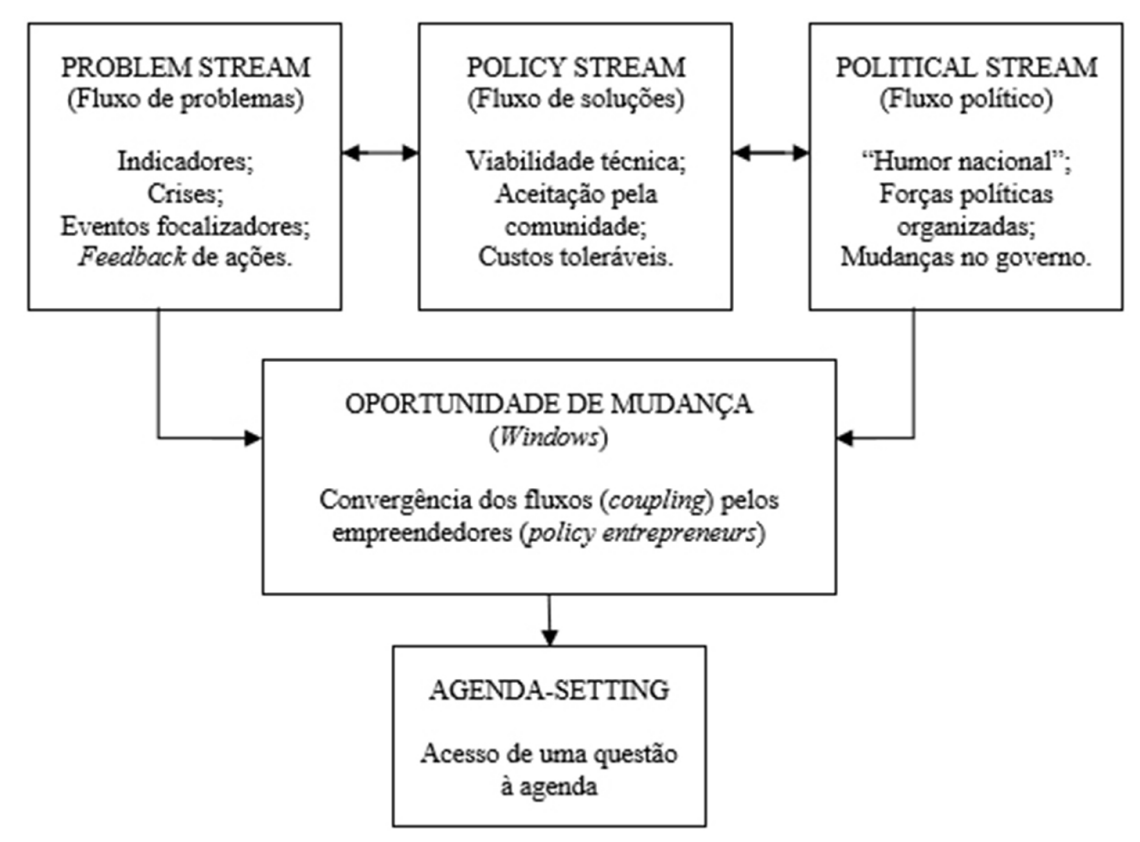

Fuente: Kingdon (2003:182)

Los tres flujos convergen en algún momento, haciendo posibles los cambios y que un problema tenga acceso a la agenda (Almeida, Silva y Silva, 2013). Además, según Gottens et al. (2013) el modelo preconiza la presencia de ambigüedad en la toma de decisiones del sector público.

\subsubsection{El problema}

En el primer flujo del modelo propuesto por Kingdon (2003) se examina de qué forma las cuestiones son reconocidas como problemas y porque determinados problemas pasan a ocupar la agenda gubernamental.

La respuesta está tanto en los medios por los cuales los actores conocen las situaciones, como en la forma en que estas son definidas como problemas. En lo que respecta a los medios para informar a los actores sobre situaciones-problemas, Kindgon (2003) los define a partir de tres mecanismos:

a. indicadores sistemáticos: muestran los problemas de una manera sistemática, puntual y contable, como costes da asistencia médica, mortalidad infantil, desempleo etc. Sirven para poder valorar la magnitud de una situación y para observar los cambios que se van produciendo en esa situación;

b. sucesos como crisis y desastres (epidemias, cuestiones presupuestarias, de seguridad etc.), que generalmente llevan a una acción inmediata;

c. por feedback sobre el funcionamiento de los programas ya existentes, o por denuncias realizadas al congreso.

Kingdon (2003) señala que existen diferencias entre situaciones y problemas. Diariamente se afrontan en las ciudades varias situaciones que causan trastornos, pero que no llegan a constituir un problema en la agenda de los gobiernos. Las situaciones pasan a constituir un problema cuando las personas deciden que hace falta hacer algo para cambiar. De ese modo una situación es definida como problema si consideramos tres circunstancias:

a. situaciones que plantean valores importantes y pasan a ser problema;

b. situaciones comparadas com las de otros países o regiones;

c. clasificación de una situación que se hace necesaria a determinado sector de la sociedad, pudiendo plantear otro problema;

Por tanto, las situaciones pueden destacarse entre los formuladores de políticas, transformándose en problemas, para posteriormente alcanzar la agenda gubernamental. Las agendas del gobierno no solo definen los problemas, también pueden hacer que desaparezcan. Kingdon (2003) define cuatro aspectos importantes en ese proceso:

a. el gobierno puede tratar el problema o no, haciendo que las personas presten atención a otras situaciones o que pierdan la confianza en la resolución, lo que hace que las personas desistan de invertir su tiempo;

b. las situaciones que llamaron la atención pueden cambiar por el cambio de indicadores o por el surgimiento de otra crisis que demande mayor atención;

c. las situaciones hacen que las personas se acostumbren al problema o creen nuevos rótulos;

d. otras situaciones surgen y dejan de lado antiguos problemas;

Para que un problema se convierta en agenda del gobierno, un conjunto de debates, competiciones y jue- 
gos de interés de diversos sectores de la sociedad deben emerger. Muchas veces el gobierno invierte recursos en las autoridades con el objetivo de convencerlas de que una situación es un problema social y, pasen, por tanto, a tenerla en cuenta en su agenda, estableciéndose de ese modo una política pública.

\subsubsection{Las soluciones}

En el segundo flujo - policy stream, Kingdon (2003) destaca en su modelo que se refiere al conjunto de alternativas y soluciones (policy alternatives) disponibles para los problemas. Kingdon (2003) considera que las ideas generadas en ese flujo no están necesariamente relacionadas a la percepción de problemas específicos. El autor afirma que las personas no necesariamente resuelven problemas, ellas generalmente crean soluciones y procuran problemas para los que puedan buscar soluciones, los cuales la mayoría de las veces no son tenidas en cuenta por los que deciden la política.

Kindgon (2003) compara la generación de alternativas y soluciones por el modelo de multiple streams con la analogía al proceso biológico de selección natural. De la misma forma que las moléculas fluctúan en lo que los biólogos llaman "caldo primitivo", el autor entiende que las ideas respecto a las soluciones son generadas en comunidades (policy communities) y fluctúan en un "caldo primitivo de políticas" (policy primeval soup). Las ideas afrontan un proceso intenso de competición, en el que algunas sobreviven intactas, otras convergen en nuevas propuestas y otras son descartadas. Es importante señalar que las ideas son analizadas, considerando algunos aspectos:

a. la viabilidad desde el punto de vista técnico y de costes, esas generalmente sobreviven;

b. si representan valores compartidos, entonces cuentan generalmente con la aceptación del público y con la receptividad de los formuladores de políticas.

Considerando los aspectos presentados, las ideas posibles constituyen las alternativas que emergen a partir de la participación en el proceso de decisión. Kingdon (2003) considera que las comunidades generadoras de alternativas (policy communities) son bastante diversificadas, lo que implica un proceso intenso y competitivo de alternativas. Esas comunidades están compuestas por especialistas - investigadores, asesores parlamentarios, académicos, funcionarios públicos, analistas pertenecientes a grupos de interés, entre otros - que comparten una preocupación sobre un área (policy area), por eso el consenso en torno a una alternativa no es tan fácil.

El consenso en torno a una propuesta viable provoca que sea difundida en diferentes foros en búsqueda de apoyo y sensibilización de los diversos actores, de las comunidades de políticas (policy communities) y del público en general, como una forma de presión y garantía de que sean consideradas en el proceso de decisión. Kingdon (2003) cree que el proceso de difusión tiene un efecto multiplicador (bandwagon), en el que las ideas se convierten en públicas y ganan cada vez más adeptos. De ese modo, el flujo de políticas (policy stream) produce una lista li- mitada de propuestas, reuniendo algunas ideas que han sobrevivido a la competición en el proceso de selección.

\subsubsection{La política}

El tercer flujo del modelo propuesto por Kingdon (2003) está compuesto por la dimensión de la política (political stream). Según el autor, tres elementos ejercen influencia sobre la agenda gubernamental:

a. el "clima" o "estado de ánimo" nacional (national mood), los participantes comparten las mismas ideas durante un período, factor que puede favorecer los cambios en la política del país. El estado de ánimo favorable crea incentivos para la promoción de algunas cuestiones, reforzando o alterando las alternativas de la agenda.

b. cambios en la arena política como resultado del proceso electoral que traen nuevos gobiernos con nuevas configuraciones en el legislativo y en toda la estructura burocrática, factor que puede contribuir al fortalecimiento de agendas ya establecidas, modificándolas radicalmente o abandonándolas por preferir otras. Kingdon considera que el inicio de un nuevo gobierno es un momento de grandes posibilidades para los cambios y la constitución de la agenda.

c. combinación de voluntad nacional con elección es importante para la formación de agenda que se estable con mayor fuerza comparada a las sugeridas por los grupos de interés.

En ese flujo, al contrario que en el anterior, de alternativas (policy stream), en que el consenso es construido basándose en la persuasión y la difusión de las ideas, en lo político (political stream) las coaliciones son construidas a partir de un proceso de transacción y negociación entre los grupos de interés y los decisores de la política.

Kingdon (2003) resalta que en un determinado momento los tres flujos anteriormente tratados convergen generando una oportunidad de cambio, haciendo que una determinada cuestión pase a formar parte de la agenda.

\subsubsection{Oportunidad de cambio a partir de la convergencia de los tres flujos: construcción de la agenda}

Kingdon (2003) explica que la oportunidad de cambio es resultado de la convergencia de los tres flujos: problemas, soluciones y política. En un determinado momento hay reconocimiento de un problema, se sugiere una solución y las condiciones de la arena política hacen posible que una determinada situación o cuestión pase a integrar la agenda.

Se comprende, por tanto, que los cambios en la agenda ocurren cuando se da la convergencia de los tres flujos. Sin embargo, el autor considera que son los flujos de problemas y de la política los determinantes para la oportunidad de cambio. El flujo de alternativa o solución definida por las comunidades, solo pasa a integrar la agenda cuando los problemas son reconocidos y políticamente se crea la oportunidad de agenda. A pesar de esa indicación, destaca que las comunidades son importantes para hacer presión de modo que una cuestión que 
conste en la agenda se fije de hecho frente a los decisores y, en ese caso, se transforme en política pública.

El proceso de convergencia de los flujos, que tiene como consecuencia la creación de la oportunidad de cambio, es posible por la ventana de oportunidad de la política, que es la oportunidad que tienen los defensores de propuestas para encaminar sus soluciones preferidas o llamar la atención para problemas especiales. Esa ventana, o sea, la oportunidad abierta a determinadas iniciativas, se presenta y permanece abierta por un determinado período de tiempo. Si no aprovechan las oportunidades, los participantes deben esperar un nuevo momento propicio en que surja la próxima oportunidad (Kingdon, 2003).

Los empresarios de las políticas (policy entrepreneurs) influyen fuertemente en ese proceso. Al defender intereses de grupos, actúan e invierten recursos de forma que puedan influir en la agenda de decisión.

El modelo de Kingdon permite la comprensión del proceso de convergencia y de ventana de oportunidad que fue creado en Brasil, para que el problema de la rotura de los embalses de desechos de minerales formara parte de la agenda de decisión en la política de actividad mineral y sus externalidades.

\subsubsection{Convergencia entre problemas, soluciones y dinámica política: las externalidades de la explotación de minerales en la agenda gubernamental en Brasil}

En este aspecto se observa que según Kingdon (2003) la incorporación en la agenda gubernamental de la cuestión de los embalses de desechos de minerales fue resultado del proceso de convergencia de los flujos: problema, solucio- nes y política, que creó la oportunidad de cambio, y por la ventana de oportunidad que se abrió con el estado de ánimo nacional, debido a la gravedad del problema, haciendo que esa externalidad integrase la agenda de decisión.

Es importante resaltar que los problemas que emergen de los indicadores y de los constantes sucesos (Kingdon, 2003), resultantes de las externalidades de la actividad de explotación de minerales en el país, que han tenido como consecuencia los daños causados a las comunidades locales, con una fuerte conmoción nacional, fueron importantes para la atención del nuevo gobierno que asumió el poder y sirve como justificación para su incorporación en la agenda.

Se considera que la cuestión determinante para que las externalidades de la actividad minera, especialmente los embalses de desechos de minerales, integre la agenda del gobierno no solo a nivel federal, sino también estadual y municipal, es la rotura del embalse de feijão, localizado en el municipio de Brumadinho, dado que hace poco tiempo, el estado de Minas Gerais sufrió también la rotura de un embalse de una empresa del grupo VALE, que destruyó la comunidad de Bento Gonçalves.

A partir de esa perspectiva analítica, se observa que la actividad de extracción de minerales desarrollada por grandes empresas, como en el caso de VALE, el papel del Estado en la regulación y control, pasa a cuestionarse por especialistas, trabajadores, comunidades locales y sectores de la sociedad civil organizada, que exigen una respuesta inmediata del Gobierno y de la empresa, para minimizar los daños y evitar que ocurran nuevos sucesos, no solamente en la región donde ha pasado, sino también, en otras regiones do Brasil donde están localizados embalses de desechos, como muestra la figura 2.

Figura 2. Embalses de desechos en Brasil (Departamento Nacional de Producción Mineral, 2018).

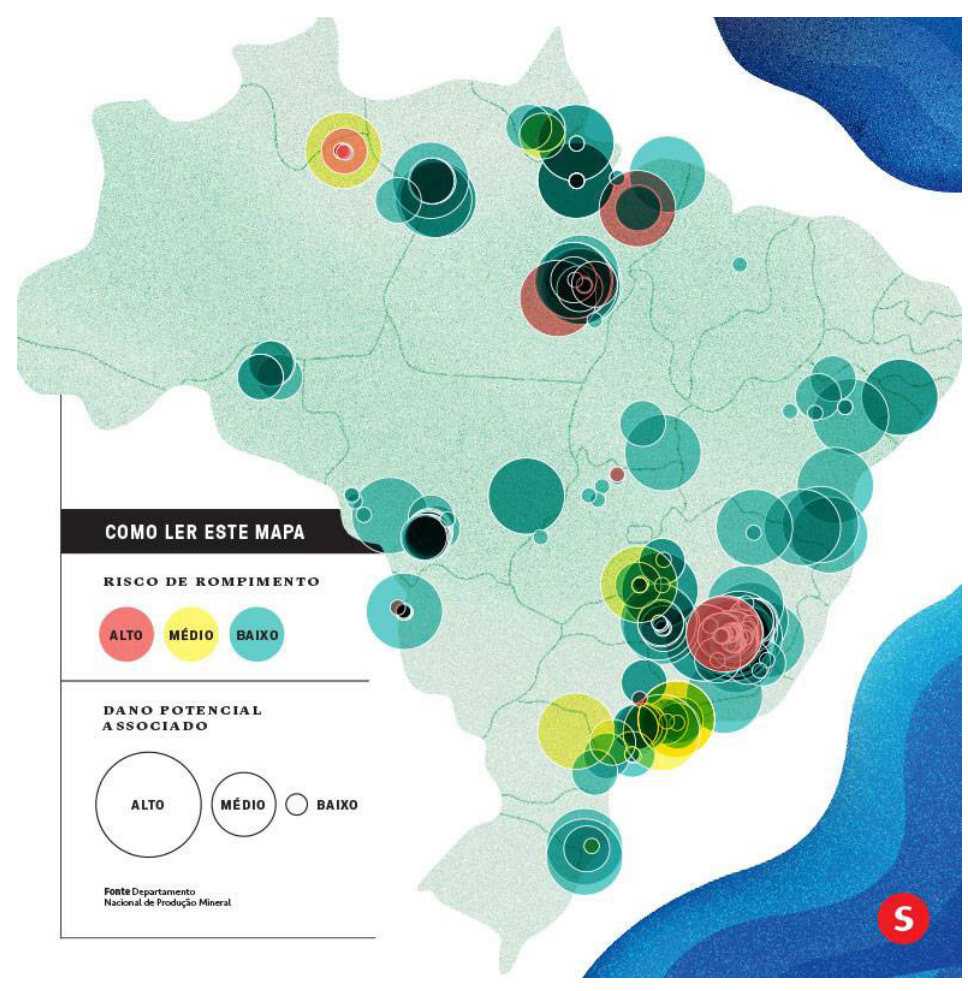

Fuente: Kingdon (2003:182). 
Varias acciones fueron realizadas por el Gobierno Federal con el fin de acompañar y fiscalizar los embalses de desechos de minerales, con efectos más allá de la región de los accidentes. Tales acciones fueron reglamentadas por actos oficiales del gobierno y establecieron las atribuciones de cada ente federado (Unión, Estados y Municipios) en la mitigación de los problemas.

El examen de tales actos por medio de los documentos, de dominio público, hizo posible la comprensión de las soluciones presentadas por el Gobierno Federal, como trata la próxima sección.

\section{Resultados y discusiones}

\subsection{Las soluciones del Gobierno para afrontar el problema}

Las soluciones para afrontar los problemas provocados por los desastres resultantes de la explotación de la actividad de minería, especialmente en las regiones afectadas, fueran establecidas por los documentos oficiales: el decreto $n^{\circ} 9.691$, de 25 de enero de 2019, resolución $n^{\circ}$ 1 , de 28 de enero de 2019, resolución n ${ }^{\circ} 2$, de 28 de enero de 2019; aprobados por el Gobierno Brasileño, como directrices para que los diversos órganos del Estado, en el nivel federal, estadual y municipal, pudiesen implementar acciones inmediatas, como forma de minimizar los daños socioambientales y humanos.

\subsubsection{El Decreto $n^{0}$ 9.691, de 25 de enero de 2019}

El Gobierno Brasileño publicó el Decreto no 9691 de 25 de enero de 2019, el cual crea el Consejo Ministerial de Supervisión de Respuestas a Desastres, con la finalidad de acompañar y fiscalizar las actividades a ejecutar a causa de la rotura del embalse de Córrego Feijão, en el municipio de Brumadinho, Estado de Minas Gerais, y de sus repercusiones en la Cuenca del Rio Paraopeba.

El Consejo Ministerial de Supervisión de Respuestas a Desastres está compuesto por los siguientes Ministros del Estado: Jefe de la Casa Civil de la Presidencia de la República (que lo coordina); de Defensa; de Ciudadanía; de Salud; de Minas y Energía; de Medio Ambiente; de Desarrollo Regional; de la Mujer, de la Familia y de los Derechos Humanos; Jefe del Gabinete de Seguridad Institucional de la Presidencia de la República y el Abogado-General de la Unión.

El referido Decreto estableció como órgano ejecutivo del Consejo, al Comité de Gestión y Evaluación de Respuestas a Desastres, como el desastre ocurrido en el embalse de Córrego de Feijão, y sus repercusiones en la cuenca del Rio Paraopeba, compuesto por un miembro de cada representante del Consejo. Ese Comité tiene como objetivo acompañar las acciones de socorro, de asistencia, de restablecimiento de servicios esenciales a los afectados y de recuperación del ecosistema.

La participación de representantes del gobierno local y de otras instituciones fue limitada por el referido Decreto, los cuales podrán ser convidados a acompañar o a participar en las reuniones del Comité de Gestión y
Evaluación de Respuestas a Desastres, pero sin derecho a voto. Tal limitación evidencia un modelo de gestión y regulación exclusivamente para el poder público de arriba para abajo (top-down), no garantizando que las decisiones del Comité tengan en cuenta los intereses reales de los actores locales.

Para que se establezca la gobernanza pública local, se cree que el modelo de abajo para arriba (bottom-up) es más democrático y participativo, pues hace posible la implicación de las partes interesadas en el proceso de las decisiones que les afectan. De hecho, como nos muestra Oliveira (2006), el ideal del proceso de planificación es el de la convergencia de los dos modelos presentados por Sabatier (1986).

\subsubsection{Resolución $n^{0} 1$, de 28 de enero de 2019}

El Consejo Ministerial de Supervisión de Respuestas a Desastres por medio de la Resolución n ${ }^{\circ} 1$, de 28 de enero de 2019 , recomendó acciones y medidas de respuesta a la rotura del embalse de Córrego de Feijão, en el Municipio de Brumadinho, Estado de Minas Gerais.

El examen de este documento desvela en sus artículos $1^{\circ}$ y $2^{\circ}$ que el Gobierno Federal recomienda a los órganos y a las entidades de la administración pública federal que sigan priorizando esfuerzos para la rápida atención de las víctimas directas e indirectas de la rotura del embalse de Córrego de Feijão, en el Municipio de Brumadinho, y sus repercusiones en la Cuenca del Rio Paraopeba, así como que movilicen recursos humanos y financieros para ese fin. Entre las acciones de apoyo financiero está la liberación del Fundo de Garantia do Tempo de Serviço (FGTS) a los trabajadores y familias víctimas de la rotura del embalse.

En el artículo $3^{\circ}$ de la resolución estudiada se observa que las acciones van más allá de la región implicada y recomienda al Consejo Nacional de Recursos Hídricos que apruebe inmediatamente una moción para solicitar a los órganos fiscalizadores, en los términos de lo dispuesto en la Política Nacional de Seguridad de los Embalses, establecida por la Ley $n^{0} 12.334$, de 20 de septiembre de 2010, más efectividad en el acompañamiento y fiscalización de los embalses existentes en todo el país.

El cuadro 1 presenta las acciones contenidas en la Resolución $n^{\circ} 1$, del 28 de enero de 2019.

Las acciones sugeridas por el Consejo Ministerial de Supervisión de Respuesta a Desastres tratan de atribuciones de nivel macro y micro, al determinar los órganos fiscalizadores federales y recomendar a los demás entes federativos que exijan de los agentes fiscalizados la actualización inmediata de sus respectivos Planes de Seguridad de Embalses, de los que habla la Ley $n^{\circ} 12.334$, de 2010, con la intención de resguardar la integridad de los trabajadores y habitantes próximos de las empresas.

\subsubsection{Resolución $n^{0} 2$, de 28 de enero de 2019}

Crea el Subcomité de Elaboración y Actualización Legislativa, con el objetivo de elaborar un anteproyecto de actualización y revisión de la Política Nacional de 
Cuadro 1. Acciones contenidas en la Resolución n 1 , del 28 enero de 2019.

I realizar inmediatamente auditorías de sus procedimientos y revisar los actos normativos que orientan la fiscalización de la seguridad de los embalses;

II mantener registro de los embalses bajo su jurisdicción, con el fin de incorporarlo al Sistema Nacional de Información sobre Seguridad de los Embalses - SNISB;

III exigir de los empresarios el cumplimiento de las recomendaciones contenidas en los informes de inspección y la revisión periódica de la seguridad;

IV exigir de los empresarios el registro y la actualización de las informaciones relativas a los embalses en el SNISB;

V realizar inmediatamente la fiscalización en los embalses bajo su jurisdicción, priorizando aquellas clasificadas como de "daño potencial asociado alto" o como "riesgo alto".

Fuente: DOU (2019).

Seguridad de Embalses. Ese Subcomité estará compuesto por un representante, titular y suplente, de los siguientes órganos y entidades de la administración pública federal: I) Casa Civil de la Presidencia de la República, que lo coordinará y le prestará apoyo administrativo; II) Ministerio de Defesa; III) Ministerio de Minas y Energía; IV) Ministerio de Medio Ambiente; V) Ministerio do Desarrollo Regional; VI) Secretaria General de la Presidencia de la República; VII) Secretaria de Gobierno de la Presidencia de la República; VIII) Gabinete de Seguridad Institucional de la Presidencia de la República; IX) Abogado General de la Unión; X) Agencia Nacional de Aguas - ANA; XI) Agencia Nacional de Energía Eléctrica - Aneel; XII) Agencia Nacional de Minas - ANM; XIII) Instituto Brasileño de Medio Ambiente y de Recursos Naturales Renovables - Ibama; y XIV) Operador Nacional del Sistema Eléctrico - ONS.

Dándoles muy pocos días de plazo para realizar cambios: 30 días desde su formación. Posteriormente el trabajo del Subcomité se someterá al Consejo Ministerial de Supervisión de Respuestas al Desastre para su aprobación en forma de informe en un plazo de cinco días útiles, contada la fecha a partir de la conclusión de sus trabajos.

El examen de esta resolución establece que representantes de otros órganos y entidades de la administración pública federal podrán ser convidados a participar de sus reuniones, aunque sin derecho a voto, evidenciado las características del modelo racionalista instrumental, cuya participación y decisión sobre las cuestiones públicas se centran en el responsable político, sin ninguna mención a la participación de representantes de gobiernos de los estados, municipales y de representantes de la sociedad civil, dado que se trata de la revisión de una política, cuyas consecuencias afectan a la vida de muchos brasileños, de ahí la importancia de oír a los actores locales ( Chambers, 1994).

\section{Conclusiones}

Infelizmente, como en muchas otras ocasiones, no son las políticas las que fallaron, sino su aplicación. La legislación brasileña es sólida, comparable, y, en algunos casos, más severa, que la de otros países del mundo. Sin embargo, existe falta de claridad sobre el papel, obligaciones y responsabilidades de cada institución en los ámbitos federal, estadual y municipal, y sobre la capacidad para controlar y fiscalizar el cumplimiento de las normas (que deben ser constantemente mejoradas, principalmente después de situaciones límites como los de esos sucesos), el tan famoso "cumplimiento". Las licencias ambientales en Brasil, por ejemplo, son desproporcionalmente burocráticas con la licencia de ciertas empresas de pequeño tamaño y bajo riesgo. Los requerimientos y la documentación van mucho más allá de la capacidad de los propios órganos públicos para procesarlos y aprobarlos, atrasando obras o simplemente dejando empresas en situación irregular. Por otro lado, falta fiscalización y control efectivo de obras y empresas de gran tamaño y riesgo, donde muchas veces intereses específicos prevalecen por encima de la ley.

El estudio en el campo teórico hizo posible comprender que se abrió una "ventana de oportunidad", como resultado de la rotura del embalse de desechos minerales en el municipio de Brumadinho y las pérdidas humanas y ambientales, por la convergencia de los flujos (problemas, soluciones, políticos) e hizo que el problema de los embalses de desechos integre la agenda del país. También influyeron las noticias en los medios de comunicación y la movilización de la población.

El modelo de Kingdon aun cuando considera la convergencia de los tres flujos para la creación de la ventana de oportunidad, no puede afirmar que en el flujo soluciones, estas sean de hecho de interés social (bottom-up). Se puede deducir un retroceso en el enfoque socio céntrico en el tratamiento de las cuestiones públicas, con las decisiones estado céntricas contenidas en los decretos y resoluciones publicadas.

En el campo empírico el examen de documentos de dominio público reveló que fueran propuestas varias acciones por el Gobierno Federal, con carácter instrumental normativo, a los estados y los municipios, para evitar que los impactos de la actividad mineral causen más daños a los trabajadores y a las comunidades locales.

Se comprobó la creación del Subcomité de Elaboración y Actualización Legislativa para elaborar un anteproyecto de actualización y revisión de la Política Nacional de Seguridad de Embalses, establecida por la Ley $\mathrm{n}^{\circ} 12.334$, de 20 de septiembre de 2010 . Aun así, se considera que el problema proviene de 
la regulación, fiscalización y control de los órganos competentes.

Señalando para concluir que los problemas de los embalses de desechos no se solucionan con la elaboración de una nueva política sino con la efectividad de la que existe y si se elabora una nueva Ley es fundamental la implicación de los actores locales en los procesos de elaboración y seguimiento de la política.

Como contribuciones futuras se sugieren estudios de seguimiento y evaluación de las decisiones tomadas al integrar el problema en la agenda (limitación del modelo de Kingdon - pre-decisión y decisión).

\section{Bibliografía}

Almeida, L.A.; Silva, A.P. y Silva, M.A. (2015).”Os múltiplos fluxos na formaçao da agenda climática em Palmas e a participação de redes transnacionais de governos locais", Teoria\&Pesquisa: Revista de Ciencia Política 22:37-49.

BrasiL. Lei $\mathrm{n}^{\mathrm{o}}$ 12.334, de 20 de setembro de 2010. Estabelece a Política Nacional de Segurança de Barragens destinadas à acumulação de água para quaisquer usos, à disposição final ou temporária de rejeitos e à acumulação de resíduos industriais, cria o Sistema Nacional de Informações sobre Segurança de Barragens e altera a redação do art. 35 da Lei n o 9.433, de 8 de janeiro de 1997, e do art. 4 o da Lei n o 9.984, de 17 de julho de 2000. Diário Oficial da União. Brasília, 21 set. 2010.

- Decreto $n^{\circ}$ 9.691, de 25 de janeiro de 2019. Institui o Conselho Ministerial de Supervisão de Respostas a Desastre e o Comitê de Gestão e Avaliação de Respostas a Desastre em decorrência da ruptura da barragem do Córrego Feijão, no Município de Brumadinho, Estado de Minas Gerais, e de suas repercussões na Bacia do Rio Paraopeba. Diário Oficial da União. Brasília, 25 jan. 2019.

- Resolução $n^{\circ} 1$, de 28 de janeiro de 2019. Recomenda ações e medidas de resposta à ruptura da barragem do Córrego do Feijão, no Município de Brumadinho, Estado de Minas Gerais. Diário Oficial da União. Brasília, 28 jan. 2019.

- Resolução no 2, de 28 de janeiro de 2019. Institui o Subcomitê de Elaboração e Atualização Legislativa, com o objetivo de elaborar anteprojeto de atualização e revisão da Política Nacional de Segurança de Barragens, estabelecida pela Lei $\mathrm{n}^{\circ} 12.334$, de 20 de setembro de 2010. Diário Oficial da União. Brasília, 28 jan. 2019.

Chambers, R. (1994) “The origins and practice of participatory rural appraisal”. World Development, 22: 953-969.

Diario Globo (2015). "Barragem se rompe, e enxurrada de lama destrói distrito de Mariana". (Consultado, el 22 de enero de 2019).

Farias, Carlos Eugênio Gomes (2002). "Mineração e meio ambiente no Brasil”. Relatório do CGEE/PNUD, 76: 2-5.

Faria, Cláudia Feres (2010). "Estado e organizações da sociedade civil no Brasil contemporâneo: construindo uma sinergia positiva”. Revista de Sociologia e Política, Curitiba, 18 (36): 187-204.

Gottems et al. (2013). "O modelo dos múltiplos fluxos de Kingdon na análise de politicas de saúde: aplicabilidades, contribuiçoes e limites", Saúde e Sociedades, 22: 511-520.

Kingdon, John Wells (2003). Agendas, Alternatives, and Public policies. Boston: Addison-Wesley Longman Inc.

Kokke, M. (2017). "El desastre ambiental del Municipio de Mariana y la construcción de un modelo de respuesta". Diario Ambiental, 163.

Lindblom, C. E. (1979). “Still muddling, not yet through”, Public Administation Review, 39: 517-526.

Losekann, C. y Mayorga C. (2018). Desastre na Bacia do Rio Doce: Desafios para a universidade e para instituições estatais. Rio de Janeiro: Folio Digital - Letra e Imagem.

Marques, E y Pimenta, C.A. (2013). A política pública como campo multidisciplinar. São Paulo: Editora UNESP.

Metter et al. (2015). "A introduçao do complexo economico industrial da saúde na agenda de desenvolvimento: uma análise a partir do modelo de fluxos múltiplos de Kingdon" Revista de Administração Pública, 49: 915-936.

Milanez, B. y Losekann, C. (Org.). (2016). Desastre no Vale do Rio Doce: Antecedentes, impactos e ações sobre a destruição. Rio de Janeiro: Folio Digital - Letra e Imagem.

Oliveira, J. A. P. (2006). “Desafios do planejamento em políticas públicas: diferentes visões e práticas”. Revista Administraçao Publica, 40 (1): 273-88.

Sabatier, P. A. (1986). "Top-down and bottom-up approaches to implementation research: a critical analysis and suggested synthesis", Journal of Public Policy, 6 (1): 21-48.

Gomes Miranda da Silva, R., Pereira Bezerra, T. C. y Borda Vilar Guimaraes, P. (2017). "A desconsideração da Pessoa Jurídica em casos de desastres ambientais: Estudo de caso do rompimento da Barragem em Mariana/MG”, Revista FIDES, 8 (30): 7-17.

Simon, R. A. (1947). Administrative behavior, a study of decision-making processes in administrative organization. New York: The Macmillan Co., p. xvi-259.

Souza, C. (2006) Estado do campo da pesquisa em políticas públicas no Brasil. Brasilea: Asociação Nacional de PósGraduação e Pesquisa em Ciências Sociais.

Tenório, F. G., y Rozenberg, J. E. (1997) “Gestão pública e cidadania: metodologias participativas em ação”, Cadernos Gestão Pública e Cidadania, 2 (7): 101-122.

Veesaert, C., Cardia, R.J. y Tsuzuki, A.L. (2005). Segurança de barragem-questões de responsabilidade. XXVI Seminário Nacional de Grandes Barragens, Goiânia (Goias), 11 a 15 de abril.

Zhouri, A. (2017). Mineração, violências e resistências: um campo aberto à produção de conhecimento no Brasil. Marabá: Iguana, dez. 